\title{
Supported online cognitive behavioural therapy for bulimia nervosa: a study protocol of a randomised controlled trial
}

\author{
Sarah Barakat ${ }^{1,2^{*}}$ D , Stephen Touyz ${ }^{1}$, Danielle Maloney ${ }^{1}$, Janice Russell ${ }^{1,4}$, Phillipa Hay ${ }^{5,6}$, Michelle Cunich ${ }^{3,7,8}$, \\ Sharyn Lymer ${ }^{3}$, Marcellinus Kim ${ }^{4}$ Sloane Madden ${ }^{9}$, Jane Miskovic-Wheatley ${ }^{1}$ and Sarah Maguire ${ }^{1}$
}

\begin{abstract}
Background: Despite the availability of effective treatments for bulimia nervosa (BN), a number of barriers to accessibility exist. Examples include access to trained clinicians, the expense of treatment, geographical limitations, and personal limitations such as stigma regarding help seeking. Self-help interventions, delivered via a digital platform, have the potential to overcome treatment gaps by providing patients with standardised, evidence-based treatments that are easily accessible, cost-effective, and require minimal clinician support. Equally, it is important to examine the shortcomings of digital interventions when compared to traditional to face-to-face delivery (e.g., high dropout rates) in order to maximise the therapeutic effectiveness of online, self-help interventions.
\end{abstract}

Methods: A three-arm, multisite randomised controlled trial will be conducted in Australia examining the effectiveness and cost-effectiveness of a newly developed online self-help intervention, Binge Eating eTherapy (BEeT), in a sample of patients with full or sub-threshold BN. The BEeT program consists of 10, multimedia sessions delivering the core components of cognitive behaviour therapy. Eligible participants will be randomised to one of three groups: independent completion of BEeT as a purely self-help program, completion of BEeT alongside clinician support (in the form of weekly telemedicine sessions), or waitlist control. Assessments will take place at baseline, weekly, post-intervention, and three-month follow up. The primary outcome is frequency of objective binge episodes. Secondary outcomes include frequency of other core eating disorder behavioural symptoms and beliefs, psychological distress, and quality of life. Statistical analyses will examine treatment effectiveness, feasibility, acceptability and cost effectiveness.

Discussion: There is limited capacity within the mental health workforce in Australia to meet the demand of people seeking treatment for eating disorders. This imbalance has only worsened following outbreak of the COVID-19 pandemic. Further research is required into innovative digital modes of treatment delivery with the capacity to service mental health needs in an accessible and affordable manner. Self-help programs may also appeal to individuals who are more reluctant to engage in traditional face-to-face treatment formats. This study will provide rigorous evidence on how to diversify treatment options for individuals with BN, ensuring more people with the illness can access evidence-based treatment.

*Correspondence: sarah.barakat@sydney.edu.au

${ }^{1}$ InsideOut Institute, Central Clinical School, The University of Sydney

Sydney Local Health District, Sydney, Australia

Full list of author information is available at the end of the article permits use, sharing, adaptation, distribution and reproduction in any medium or format, as long as you give appropriate credit to the original author(s) and the source, provide a link to the Creative Commons licence, and indicate if changes were made. The images or other third party material in this article are included in the article's Creative Commons licence, unless indicated otherwise in a credit line to the material. If material is not included in the article's Creative Commons licence and your intended use is not permitted by statutory regulation or exceeds the permitted use, you will need to obtain permission directly from the copyright holder. To view a copy of this licence, visit http://creativecommons.org/licenses/by/4.0/. The Creative Commons Public Domain Dedication waiver (http://creativeco mmons.org/publicdomain/zero/1.0/) applies to the data made available in this article, unless otherwise stated in a credit line to the data. 
The study has been registered with the Australia New Zealand Clinical Trials Registry (ANZCTR Registration Number: ACTRN12619000123145p). Registered 22 January 2019, https://www.australianclinicaltrials.gov.au/anzctr/trial/ACTRN 12619000123145.

Keywords: Feeding and eating disorders, Online healthcare, Cost-effectiveness, Cognitive behaviour therapy, Selfhelp treatment, COVID-19 driven demand for online care

\section{Introduction}

Bulimia nervosa $(\mathrm{BN})$ is an eating disorder $(\mathrm{ED})$ characterised by recurrent objective binge episodes followed by behaviours intended to compensate for the food eaten during the binge [1]. Binge episodes must involve the consumption of an objectively large amount of food and be accompanied by a sense of lack of control [1]. BN is one of the most common types of EDs globally [2]. According to the Global Burden of Disease Study, the global prevalence rates of $\mathrm{BN}$ in 2019 was estimated to be 176.2 per 100,000 people [2]. Epidemiological research conducted on an Australian sample from 1998 to 2008 has identified an upward trend in core symptoms BN including objective binge episodes and extreme dieting [3]. These findings are concerning given the significant physical and psychological impairments imposed by $\mathrm{BN}$ [4]. Individuals with $\mathrm{BN}$ have been found to report greater functional impairment, higher emotional distress, and lower quality of life than their age-matched peers without the illness [5, 6]. Additionally, significant medical complications are often incurred by individuals with BN as well as economic costs as a result of lower labour force participation, greater absenteeism, and higher healthcare costs $[2,7,8]$. The impacts of the illness are only worsened by personal barriers of shame and stigma associated with disordered eating behaviours, which function to discourage help seeking and result in worsening of untreated symptoms [9]. Recent research has identified an average delay of 8.4 years between the onset of $\mathrm{BN}$ symptoms and the first instance of help seeking behaviours, with stigma reported as the most impactful barrier [10].

Individual psychological therapy is recognised as the first-line treatment for adults with BN [11], with cognitive behavioural therapy (CBT) having the strongest evidence base of all therapeutic modalities [12-14]. Unfortunately, on average only $23.2 \%$ of individuals with an ED access an evidence-based treatment [15]. Limited availability of trained clinicians and poor scalability of therapist-led CBT have been identified as contributors to the poor translation of evidence-based research to realworld populations [16]. The capacity of clinical services to meet the demand of $\mathrm{BN}$ patients is often limited by the structure of therapist-led CBT, with most evidence-based treatments stipulating 20 hours of face-to-face therapist contact across a 20 -week period $[17,18]$. Although there is emerging evidence to suggest ten-session therapistled CBT may produce similar clinical outcomes for BN [19], the predicament of limited treatment accessibility remains for those residing in rural and remote areas with very few ED trained clinicians operating outside of metropolitan regions of Australia [20]. Given the demands on public health resources, those who are forced to seek treatment within the private sector are faced with a significant financial expense [21]. There are also concerns regarding the quality of CBT being delivered in the community with studies reporting that as few as $6 \%$ of clinicians adhere to evidence-based manuals [22].

Greater attention must be directed towards adapting evidence-based treatments to fit within more timeand cost-efficient models of healthcare delivery, thereby improving accessibility and fidelity within resource constraints. As part of a stepped care model, lower intensity treatments, such as online self-help programs, can be offered to those with less severe clinical presentations in order to prioritise specialist therapeutic skill for patients with greater symptom complexity. Technology-based interventions allow for numerous patients to simultaneously engage with standardised, evidence-based treatments at any time and location that best suits them [16, 23]. The anonymity of digital interventions may assist with the shame and secrecy experienced in relation to bulimic behaviours and the seeking of treatment [24]. Importantly, the recent COVID-19 pandemic has emphasised the need for a technology revolution in the mental health care system [25-30]. Fortunately, digital platforms have allowed mental health services to meet the needs of patients in lieu of in-person contact, highlighting the convenience and flexibility of online treatments and their capacity to overcome the shortcomings of traditional treatment delivery in the current pandemic situation $[26$, $27,31,32]$.

Several systematic reviews have concluded that digital self-help interventions are effective in reducing eating disorder behaviours [33-40]. There is evidence to also suggest that the treatment outcomes of self-help interventions are significantly enhanced by the addition of low-intensity support from a clinician, such as a fortnightly, check-in email or phone call [12, 39, 41-43]. Supported self-help interventions only demand one-fifth 
of the therapist contact hours required for a complete CBT course, meaning they can be delivered in settings with low resources and capacity limits [42]. The provision of clinician support as an adjunct to self-help programs has been suggested as a viable solution to one of the most powerful critiques of digital interventions, that being the high rates of non-adherence [37, 38]. Internetdelivered CBT has the highest dropout rates of all ED treatments, ranging up to $47.2 \%$ [44]. Given the potential impact of non-adherence upon the therapeutic outcomes for patients, several reviews have highlighted the need for ongoing development and examination of novel digital treatments, with the aim of reducing the discrepancy in adherence between online platforms and more traditional modes of face-to-face treatment delivery $[35,37$, 38].

Two large published studies provide evidence for the use of online CBT-based, self-help programs for individuals with $\mathrm{BN}$. As part of a randomised controlled trial (RCT) conducted by Sánchez-Ortiz et al. [45] significant improvements were reported in a sample of 75 patients with full or subthreshold $\mathrm{BN}$ following completion of an eight-session online CBT-based program. Abstinence rates were comparable to those of patients receiving a manual-based CBT self-help intervention and face-toface CBT program [46, 47]. Similarly, Pretorius et al. [48] observed significant reductions in $\mathrm{BN}$ symptomology in response to an online CBT program, supplemented with email support from a clinician and peer support via message boards, which were maintained at six-month follow up. An abridged version of BEeT has also been successfully piloted proving its acceptability, feasibility, and effectiveness in reducing core symptoms of BN [49].

Despite the documented effectiveness of online therapies for ED, the availability and uptake of evidence-based digital interventions in the public has been minimal [50]. Very few of the digital interventions examined in preceding clinical trials uphold the technological sophistication required to support user engagement. For example, very few are available as a smartphone application or employ interactive and personalised functions [35]. Further, the vast majority of ED smartphone applications available in the commercial marketplace have not been developed or evaluated by clinical researchers and provide advice that is not informed by evidence-based approaches [51]. Additionally, to our knowledge none of the current online therapies for EDs are available in Australia, with all programs having been developed and evaluated in the USA, UK, and European countries [35]. Consequently, the pilot study of BEeT is the only known evidence which supports the effectiveness of these technologies within the Australian population [49]. Considering the increasing demand for by-distance treatments in the aftermath of COVID-19, and in order to ensure evidencebased treatments are developed in a timely manner, these questions warrant examination. The Binge Eating eTherapy (BEeT) program was developed to address the need for online CBT-based, self-help programs for BN in Australia and to meet the demand for online therapies with up-to-date digital features which can be safely accessed by the patient themselves.

\section{Study objectives}

This randomized controlled trial will compare three study conditions: (1) independent completion of BEeT as a pure self-help program; (2) completion of BEeT alongside 30-min, weekly clinician support; and (3) waitlist control condition (WLC).

The aims of this current trial are to:

1. Investigate the effectiveness of clinician-supported BEeT compared to pure self-help BEeT and WLC in bringing about significant symptom reduction in individuals with $\mathrm{BN}$ or subthreshold $\mathrm{BN}$;

2. Examine site-specific outcomes of treatment implementation of clinician-supported BEeT as a lowintensity, eating disorder treatment intervention within the NSW health system; and

3. Determine the cost-effectiveness of WLC as compared to both pure self-help BEeT and clinician-supported BEeT.

\section{Methods}

The present study protocol is reported according to the SPIRIT checklist [52].

\section{Trial design and setting}

This study is a multisite, three-arm randomized controlled trial inclusive of two active interventions and a WLC. Participants will be recruited from the general community in Australia via advertisements on health websites and social media, referrals made from health professionals, and paper advertisements in mental health and medical clinics. If considered eligible following a screening call, the participant will be instructed to complete a standardised medical assessment with their general practitioner (GP) prior to entry to the study. After providing written informed consent, participants will be randomised to either the WLC condition or one of the intervention conditions, including pure self-help BEeT or clinician-supported BEeT. The intervention is entirely via digital modalities, including (1) online eTherapy platform and (2) secure videoconferencing platform (only for participants receiving clinician-supported BEeT). Participants randomised to the clinician-supported BEeT 
condition will engage in telemedicine sessions delivered by clinicians based at one of the following sites: specialist eating disorder outpatient clinic, adult community mental health team, university-based research team, and youth mental health clinic (headspace). All sites are based within New South Wales, Australia. See "Appendix A" for a list of study sites. Participants will be assessed at baseline (T0), weekly, post-intervention (T1: 12 weeks), and at 3-month follow-up (T2). The study commenced in May 2020. The study protocol has been approved by a Human Research Ethics Review Board (HREC \#X180486). See Fig. 1 for an overview of the study process.

\section{Eligibility criteria}

The primary inclusion and exclusion criteria are listed in Box 1. In addition to these, all participants will be required to complete a standardised medical assessment with their general practitioner (GP) prior to entry in the study. Participants must return clearance form signed by the GP, confirming that they are medically fit to participate, and that the GP agrees to monitor the medical and psychiatric stability of the participant throughout the trial at intervals they deem to be clinically appropriate.

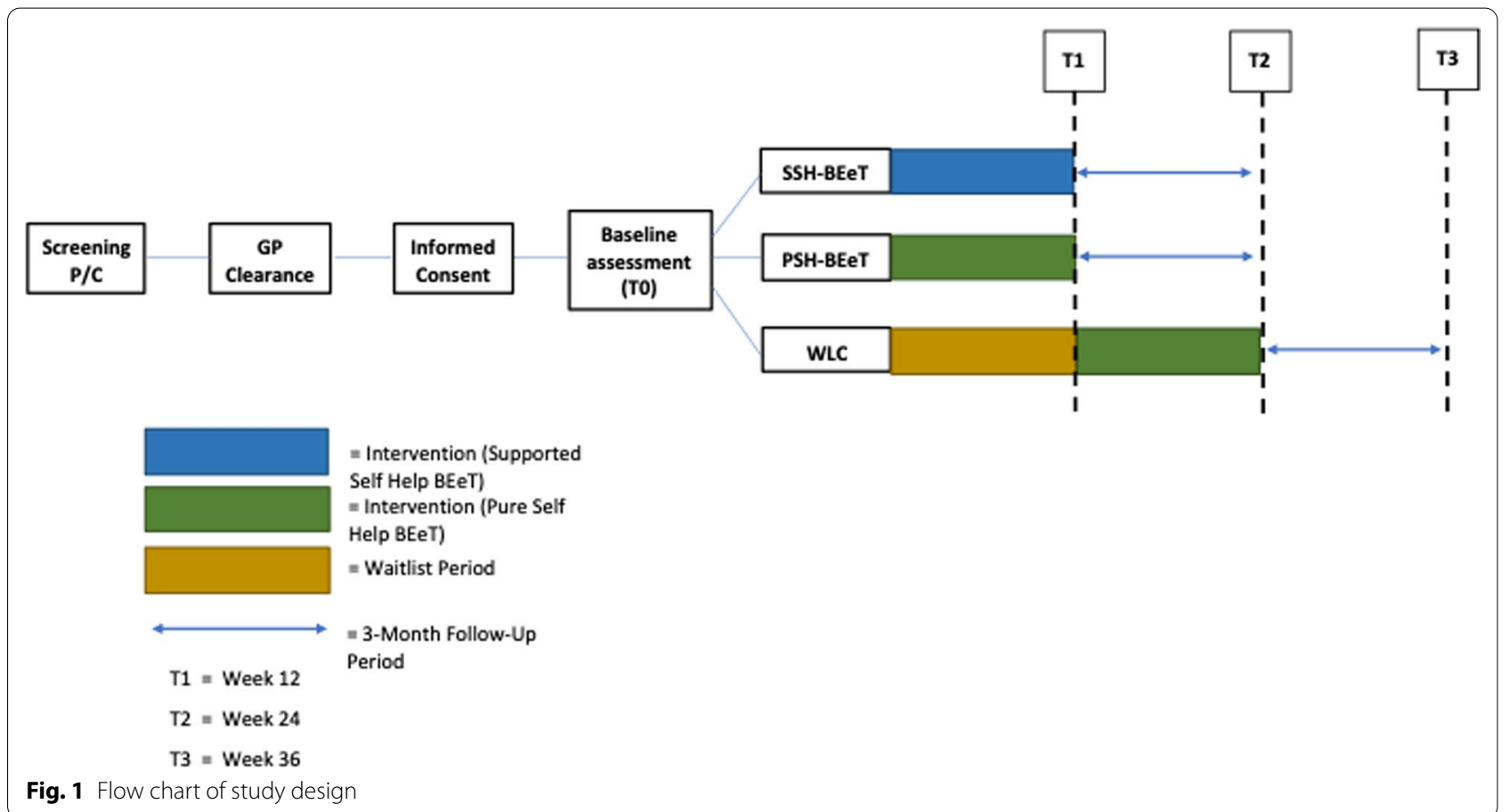

Box 1 Eligibility criteria for participation in trial

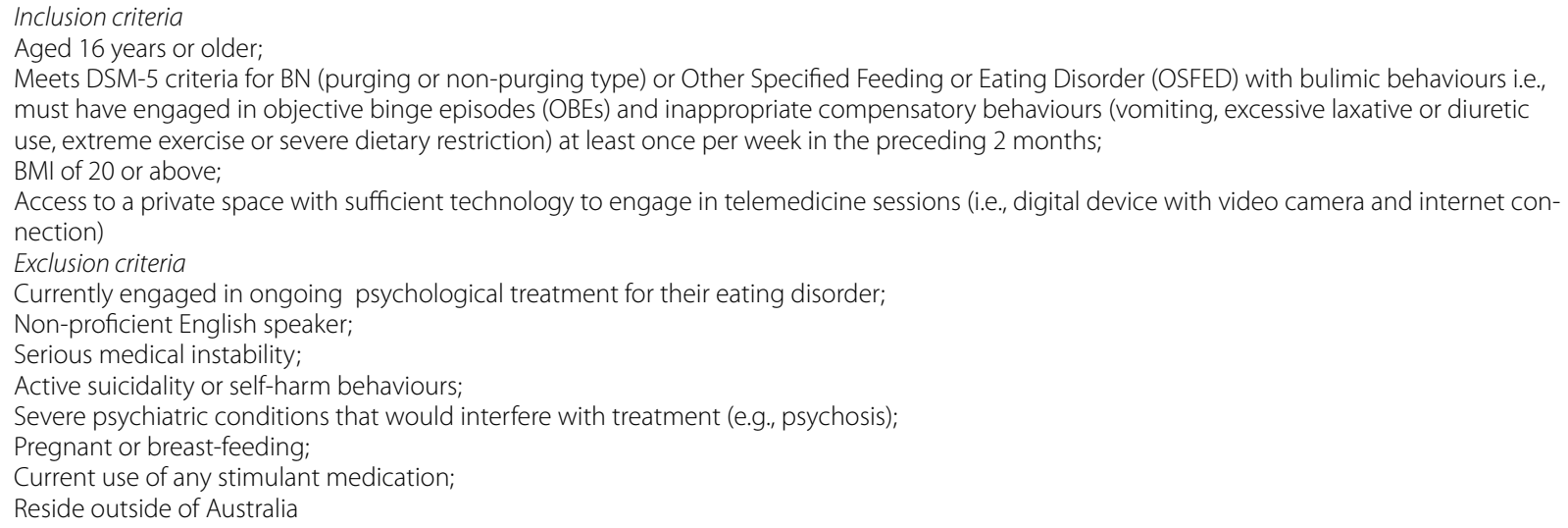




\section{Interventions}

\section{Binge Eating eTherapy (BEeT)}

All participants will receive access to BEeT, including both intervention conditions and WLC condition (post 10 -week waitlist period). BEeT is an online self-help program that delivers the core components and strategies of CBT proven to be effective in treating eating disorders. BEeT is a 10-week program which consists of 10 , one-hour interactive multimedia sessions. BEeT aims to decrease binge eating episodes and compensatory behaviours through the introduction of behavioural techniques which work to establish structured, regular eating patterns as well as cognitive techniques designed to challenge unhelpful thinking patterns related to one's weight and appearance (See Table 1 for outline of the program components). Users are guided through the online sessions by pre-recorded videos of a therapist. Also included within the sessions are interactive activities to assist users in gaining a personalised understanding of key CBT skills. Each session becomes available every seven days, given that the previous session has been fully completed. Additionally, the program contains an inbuilt digital calendar to promote real-time self-monitoring practice via their digital device using tools such as food diary monitoring, thought challenging, feared foods exposure, and meal planning. The self-monitoring tools uphold several interactive features including a daily or weekly goal setting, scheduled reminders of goals or planned mealtimes, labelling of thoughts and emotions using animated text and images, reminders of coping skills practice, prompting questions to assist with re-framing thoughts, and a visual overview of weekly logs using colour coding to assist with recognising patterns in eating behaviours and thoughts.

\section{Pure self-help BEeT}

Participants allocated to the pure self-help BEeT treatment group will be given access to BEeT as described following after their baseline assessment.

\section{Clinician-supported BEeT}

Participants allocated to the clinician-supported BEeT treatment group will receive access to BEeT in addition to 10 , weekly 30 -min sessions with a support clinician delivered via a videoconferencing platform. The participant will be instructed to complete each weekly online BEeT session prior to attending the associated telemedicine support session.

The telemedicine sessions adopt the following structure:

1. General check-in regarding participants' wellbeing (e.g., mood, anxiety, sleep), their eating disorder

Table 1 Overview of binge eating etherapy session content

\begin{tabular}{|c|c|c|}
\hline Session & Focus & Key principles \\
\hline Session 1 & Formulation and monitoring eating & $\begin{array}{l}\text { Psychoeducation about cognitive-behavioural therapy } \\
\text { Introduction to self-monitoring of eating behaviours }\end{array}$ \\
\hline Session 2 & Eating regularly and planning ahead & $\begin{array}{l}\text { Development of personalised case formulation } \\
\text { Psychoeducation about the role of restricting food and starvation } \\
\text { Regular eating }\end{array}$ \\
\hline Session 3 & Addressing binges & $\begin{array}{l}\text { Key strategies and skills to overcome binge eating and cope with urges to binge } \\
\text { The role of triggers in the Binge Eating Cycle }\end{array}$ \\
\hline Session 4 & Problem solving and motivation & $\begin{array}{l}\text { Problem solving } \\
\text { Motivational enhancement strategies: pros and cons of change }\end{array}$ \\
\hline Session 5 & Understanding and noticing thoughts and feelings & $\begin{array}{l}\text { Psychoeducation about emotions and unhelpful thinking patterns } \\
\text { Emotion regulation skills } \\
\text { Introduction to self-monitoring of unhelpful thoughts }\end{array}$ \\
\hline Session 6 & Coping with thoughts and feelings & $\begin{array}{l}\text { Mid-treatment reflection upon strengths and progress } \\
\text { Thought challenging }\end{array}$ \\
\hline Session 7 & Exposure challenges: Feared foods and food rules & $\begin{array}{l}\text { Introduction to feared foods and food rules } \\
\text { Development of personalised exposure hierarchy } \\
\text { How to challenge feared foods and food rules using 'Exposure Tool' }\end{array}$ \\
\hline Session 8 & Exposure challenges: Body image & $\begin{array}{l}\text { Psychoeducation about body image } \\
\text { Identification of exposure tasks to overcome checking and/or avoidance } \\
\text { Urge surfing }\end{array}$ \\
\hline Session 9 & Self-compassion and identifying values and strengths & $\begin{array}{l}\text { Strategies to foster greater self-compassion and body acceptance } \\
\text { Identifying personal values and strengths external to eating, weight, and shape }\end{array}$ \\
\hline Session 10 & Review and relapse prevention & $\begin{array}{l}\text { Relapse prevention strategies } \\
\text { Reflection upon progress and changes in symptoms across treatment } \\
\text { Discussion of other treatment options and support }\end{array}$ \\
\hline
\end{tabular}


symptoms (e.g., improved, worsened), and significant events that may have occurred since previous session.

2. Review participants' understanding of the content introduced in the previous online BEeT session.

3. Review participants' attempts to complete the learning tasks in their own time, with a particular focus upon debriefing the self-monitoring exercises.

4. Review weekly weigh-in.

5. Preparation for completion of the next online BEeT session.

All clinicians will have completed an online training course in how to deliver a supported self-help CBT intervention for $\mathrm{BN}$, which outlines session by session what needs to be addressed in the support sessions. The clinicians will be existing staff of the service and may have various health qualifications, such as psychology, social work and dietetics. It is expected that participants will meet with their support clinician for a total of approximately five hours across the entire program.

\section{Waitlist control}

Participants in the WLC will be offered the pure self-help BEeT intervention after a waiting period of ten weeks (T1). During the waiting period they will complete 10, brief weekly psychological assessments (approx. $5 \mathrm{~min}$ in length) to monitor eating disorder symptoms, psychological distress and suicidal risk.

\section{Discontinuation criteria}

Participants will be discontinued from the trial if a high level of medical or psychiatric risk is indicated. This includes severe mood instability requiring inpatient hospital admission (e.g., acute suicidality or deliberate selfharm), severe medical complications as confirmed by GP, or if the participant's BMI drops below 19. Participants will also be discontinued if they are considered to have disengaged from the intervention evidenced by three weeks of non-completion of the online BEeT session or three consecutive missed telemedicine sessions (only for clinician-supported BEeT group).

\section{Adherence strategies}

The BEeT platform contains in-built reminders to prompt engagement with the intervention. These include email notifications to alert participants to when a new online BEeT session is available and an automatic text message at approximately 6 p.m. to participants who have not made a food monitoring entry in the previous 24-h period. In addition, participants will be able to create their own personalised reminders specific to their weekly goals.

Additional efforts to re-engage participants in the pure self-help BEeT or WLC groups will consist of written contact via email to the participant after one week of disengagement, a phone call from researchers or clinicians after two weeks consecutive weeks of disengagement, and a letter to the participant and GP after three consecutive weeks of disengagement. The participant will be given an additional two weeks to reengage before they are discontinued from the trial.

\section{Contaminant care}

Participants will be permitted to seek regular consultation with other health professionals (e.g., dietitian, psychiatrist) whilst actively engaged in the trial, regardless of arm. Concurrent psychological treatment will only be permitted if it is addressing co-occurring mental health difficulties (e.g., anxiety and low mood) and not the eating disorder. Anyone receiving psychological treatment for their ED will be ineligible for the trial.

\section{Outcomes}

Data will be collected via online self-report assessments. Table 2 provides an overview of assessment points and outcome measures.

\section{Primary outcome}

The primary outcome is frequency of objective binge episodes as measured by the eating disorder examination questionnaire (EDE-Q) [53]. EDE-Q is a 30-item self-report questionnaire used to assess frequencies of core ED behaviours, including binge episodes and compensatory behaviours, and attitudinal aspects of ED psychopathology across the preceding 28 days. The EDE-Q upholds good reliability (Cronbach's $\alpha=0.90$ ) [54].

\section{Secondary outcome measures \\ Measures of eating disorder psychopathology:}

$E D E-Q S$ A shorter 12-item version derived from the EDE-Q, the eating disorder examination questionnaireshort (EDE-QS) assesses ED behaviours and psychopathology severity in the preceding seven days [55]. The EDE-QS is psychometrically and conceptually sound and has good reliability (Cronbach's $\alpha=0.90)[55,56]$.

EDQOL The eating disorder quality of life questionnaire (EDQOL) is a disease-specific measure of healthrelated quality of life [57]. The EDQOL contains 25-items which assess impairment of the ED upon four primary domains: psychological, physical/cognitive, financial, and work/school. The EDQOL has good reliability (Cronbach's $\alpha=0.90$ ) [57]. 
Table 2 Schedule of enrolment, interventions, and assessments according to SPIRIT

\begin{tabular}{|c|c|c|c|c|c|c|c|c|}
\hline & \multirow[t]{3}{*}{ TIMEPOINT** } & \multicolumn{7}{|c|}{ STUDY PERIOD } \\
\hline & & \multirow{2}{*}{$\begin{array}{l}\text { Enrolment } \\
-T_{1}\end{array}$} & \multirow{2}{*}{$\begin{array}{l}\text { Allocation } \\
0\end{array}$} & \multicolumn{5}{|c|}{ Post-allocation } \\
\hline & & & & $\mathrm{T}_{0}$ & $\begin{array}{l}\text { Weekly } \\
\text { monitoring }\end{array}$ & $\mathrm{T}_{1}$ & $\mathrm{~T}_{2}$ & $\mathrm{~T}_{3}$ \\
\hline \multirow[t]{4}{*}{ Enrolment } & Eligibility screen & $x$ & & & & & & \\
\hline & GP Clearance & $x$ & & & & & & \\
\hline & Informed consent & $x$ & & & & & & \\
\hline & Allocation & & $x$ & & & & & \\
\hline \multirow[t]{3}{*}{ Interventions } & Clinician-supported BEeT & & & 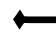 & & & & \\
\hline & Pure self-help BEeT & & & $\hookleftarrow$ & & & $\rightarrow$ & \\
\hline & WLC & & & $\hookleftarrow$ & & & & \\
\hline \multirow[t]{15}{*}{ Assessments } & Demographics & & & $x$ & & & & \\
\hline & General mental health & & & $x$ & & & & \\
\hline & Self-harm and suicidality risk assessment & & & $x$ & $x$ & $x$ & $x$ & $x$ \\
\hline & Eating disorder examination questionnaire (EDE-Q) & & & $x$ & & $x$ & $x$ & $x$ \\
\hline & Eating disorder examination questionnaire-short (EDE-QS) & & & & $x$ & & & \\
\hline & Kessler psychological distress scale (K10) & & & $x$ & $x$ & $x$ & $x$ & $x$ \\
\hline & Eating disorder quality of life questionnaire (EDQOL) & & & $x$ & & $x$ & $x$ & $x$ \\
\hline & Three-factor eating questionnaire (TFEQ) & & & $x$ & & $x$ & $x$ & $x$ \\
\hline & Young schema questionnaire & & & $x$ & & $x$ & $x$ & $x$ \\
\hline & Body mass index (BMI) & & & $x$ & $x^{1}$ & $x$ & $x$ & $x$ \\
\hline & European quality of life- 5 dimensions-5 levels (ED-5D-5L) & & & $x$ & & $x$ & $x$ & $x$ \\
\hline & Evaluation of external health services utilised & & & $x$ & & $x$ & $x$ & $x$ \\
\hline & Implementation surveys & & & & & $x$ & & \\
\hline & Working alliance inventory-short revised (WAI-SR) ${ }^{2}$ & & & & & $x$ & & \\
\hline & $\begin{array}{l}\text { Working alliance inventory for online interventions-short } \\
\text { form (WAI-TECH-SF) }\end{array}$ & & & & & $X$ & & \\
\hline
\end{tabular}

${ }^{* *} \mathrm{~T}_{0}=$ baseline assessment, $\mathrm{T}_{1}=12$ week assessment (post-intervention), $\mathrm{T}_{3}=24$ week assessment ( 3 month follow up), $\mathrm{T}_{3}=36$ week assessment (for WLC only)

${ }^{1} \mathrm{BMI}$ measured in fourth week of participation. Also measured weekly from fifth to tenth week for participants where rapid weight loss is indicated

${ }^{2}$ Only administered to clinician-supported BEeT participants

TFEQ The three factor eating questionnaire (TFEQ) is a 51-item self-report assessment which measures three dimensions of eating behaviour: cognitive restraint of eating, disinhibition/loss of control, and hunger [58]. The TFEQ has been shown to have good reliability across all three dimensions [58].

\section{Measures of general mental health psychopathology}

K10 The Kessler Psychological Distress (K10) is a 10-item self-report questionnaire used to monitor the degree of psychological distress [59]. There is evidence to support the reliability of the $\mathrm{K} 10$ as a measure of negative emotionality $[60,61,62]$.

YSQ-S3 The young schema questionnaire-short form (YSQ-SF) is a self-report questionnaire which assesses early maladaptive schemas thought to contribute to underlying dysfunctional beliefs and associated psychopathology [63].
EQ-5D-5L The five-dimensional European Quality of Life instrument (EQ-5D-5L) will be used to measure the impact of illness upon participants' mobility, self-care, usual activities, pain/discomfort, and anxiety/depression as well as an overall health score [64]. Utility weights will be obtained and used, along with life years, to calculate Quality Adjusted Life Years (QALYs).

\section{Other measures regarding the intervention}

WAI-SR The working alliance inventory-short revised (WAI-SR) is a 12-item self-report assessment which examines the degree of therapeutic alliance experienced by the patient with the clinician across three subscales: bond, task, and goal [65]. It is a widely used measure with good psychometric properties [66].

WAI-TECH-SF Working alliance inventory for online interventions-short form (WAI-TECH-SF) is adapted from the WAI-SR and aims to assess the degree of therapeutic alliance between the patient and internet-based 
self-help programs [67]. It is also a self-report measure with 12-items and has been shown to be a reliable questionnaire [67].

\section{Demographics}

Demographic characteristics will be collected using a self-designed questionnaire. These include age, gender, occupation, marital status, level of education, income, cultural background/ethnicity, and setting of residence.

\section{General mental health}

A self-designed questionnaire will collect information regarding the participant's perception of their current primary and secondary mental health concern (e.g., eating/weight issues, anxiety, depression etc.), history of mental health difficulties, and treatment status (e.g., type of mental health service and treatment being accessed).

\section{Self-harm and suicidality risk assessment}

Participants' history of suicidal and self-harming thoughts or behaviours will be assessed using a selfdesigned questionnaire. It measures severity of suicidality (e.g., suicidal thoughts, suicide attempts etc.) and deliberate self-harm (e.g., urges to self-harm, self-harm actions) in the previous 12 months to 28 days (pre-post treatment and follow up assessments) and previous seven days (weekly assessments).

\section{Participant evaluation of external health services utilised}

This purpose-built survey will capture information regarding the healthcare resources used (to calculate costs) of participants. Participants will be asked to report the number of health practitioners they have visited in the previous 28-days, including the type/speciality (e.g., GP, psychiatrist, dietitian, physiotherapist etc.), the number of visits/appointments, and the cost per visit.

\section{Implementation questionnaire}

Self-designed questionnaires will be used to assess treatment implementation from the perspective of participants, clinicians delivering supported $\mathrm{BEeT}$, and trial site managers. The outcome variables include acceptability, adoption, appropriateness, feasibility, fidelity, and sustainability [68]. Feedback will be sought from participants regarding their experience of using the $\mathrm{BEeT}$ intervention and clinicians will be asked about their perception of the suitability of the supported BEeT program for patients in their service. Site managers will be asked questions to assess their willingness to embed a supported selfhelp program, such as BEeT, within their clinical service. Question responses include a combination of 5-point Likert scale and checkbox answers.

\section{Compliance}

Measures of compliance will be monitored via the online platform on which the BEeT intervention is hosted. These include the number of self-monitoring entries logged per day, the amount of time taken to complete each online BEeT session, and the time between completion of each BEeT session. The compliance data will be included in statistical analyses of intervention response to determine their effects on key clinical and health economic outcomes.

\section{Participant timeline}

See Fig. 1 for the individual participant timeline.

\section{Sample size}

The power calculation is based on EDE-Q outcome data from a recent pilot study of BEeT [49]. The pre- to posttreatment change in objective binge episode frequency, rather than the frequency of purging was chosen as the basis for the power analysis given that not all of the participants in the current study will have engaged in purging behaviours. Accounting for multiple comparisons in the testing procedure, an alpha of 0.05 was adjusted to 0.017 under the Bonferroni method. Using a minimum of 14.9 and maximum of 23.7 for the group means (based on the findings of Barakat et al. [49]), and assuming an adjusted significance level of 0.017, a power of 0.8 , and an effect size Cohen's $\mathrm{f}=0.3$, a sample size of 48 participants is required in each of the three conditions. By applying a dropout rate of $16 \%$ (based upon findings of Barakat et al. [49]), 55 participants are needed in each group. Due to potential resourcing and time limitations, consideration was given to a worstcase scenario of sample size to be recruited. Given this, simulations of the possible impact of reduced sample size was analysed varying the parameters of an effect size (0.25-0.34) and alpha (0.01-0.025). Our worst case scenario indicates that with an effect size of 0.2 , alpha of 0.017 , and a sample size of 99 participants recruited, the study will have a power of 0.6. This sample size is supported by previous RCTs on eTherapies for eating disorders which have yielded very similar values in their power analyses [69-71]. The power analysis was calculated using STATA (Version 15) [72].

\section{Randomisation}

Randomisation will be performed independently by the National Health and Medical Research Council Clinical Trials Centre, Sydney Australia (NHMRC CTC). The allocation sequence is a permuted block list consisting of blocks randomly varying between size 6 and 9. The study group allocation will be revealed to the 
researcher via the NHMRC CTC telephone randomisation service. No stratification factors will be used.

\section{Blinding}

It is not possible to blind participants in this trial given that the difference between the two interventions and the waitlist groups can be detected easily. Blinding of outcome assessors is not applicable given all assessments consist of self-report measures. Data analysts will be blinded to the group allocation of participants.

\section{Data management and data monitoring}

Data collection will be conducted online via two digital platforms: (1) the BEeT intervention platform and (2) RedCAP, a secure electronic data capture platform. All electronic data and confidential study materials, such as participant consent forms, will be stored in an online database via the secure digital platform on which they were collected. Both platforms will only be accessible via a firewall protected website which requires a preauthorised login and password known only to the Chief Investigator and Associate Investigators involved in data collection/analysis. The BEeT website is hosted on an isolated server, which provides complete privacy and locks the database from the public internet. Data will be deidentified and stored as an SPSS datafile on an enterprisegrade storage platform provided by the University of Sydney. Each participant will be identified in the data set by a unique numerical code. Raw data will remain available on the secure digital platform and output entered routinely into the SPSS datafile as the study progresses.

An audit of files will be conducted regularly to ensure completeness and accuracy of data collection. An independent study monitor is not appointed for this study. The trial monitoring plan is implemented by staff at the University of Sydney. Participant records are selected at random to conduct source data verification on parameters such as demographic data, key outcome variables, informed consent, indicators of risk, and adverse events. Each site will have an on-site monitoring visit at least once per year during the active phase of the study. Members of the steering committee will meet quarterly to discuss any concerns or issues which emerge from the clinical trial monitoring procedures and actions and resolutions are documented.

\section{Risk management}

We anticipate very minimal to no risks to the participant using this intervention. However, it is possible that there may be a slight increase in psychological discomfort during or after completion of the online sessions. To monitor this, participants' psychiatric risk status will be tracked for the duration of the trial using weekly short psychological assessments. If suicidal thoughts or selfharm is reported, a researcher will contact the participant to complete a risk assessment and provide support as required to ensure their safety. In addition, participant's weight will be collected at two assessment points (T0 and week four) to monitor medical risks associated with rapid weight loss. If rapid weight loss is indicated, the participant will be asked to provide a weekly measure of their weight for the duration of the trial. Participants will be discontinued from the trial if their BMI is below 19. The GP will also be alerted via email if psychiatric risk rapid or weight loss is identified.

\section{Statistical methods}

Initial exploratory analysis will be conducted to ensure baseline comparability between the two intervention groups and across sites. An intention to treat analysis will be conducted. Multiple imputation analysis will be implemented in accordance with missingness assumptions that fit the data (missing at random vs missing not at random), followed by a sensitivity analysis where appropriate. Generalised linear mixed modelling (GLMM) will be used to compare the effectiveness of pure self-help BEeT versus clinician-supported BEeT vs. WLC, collapsed across settings, using change in objective binge episode frequency as the primary outcome. Co-variates in the model will include baseline information of participant age, illness severity and length of illness. This will allow for assessment of the impact of participant characteristics on the key outcomes. Changes in secondary outcomes, such as psychological distress and quality of life, will also be analysed using a GLMM framework.

Time-to-event statistical methods, in particular survival analysis (cox proportional hazard modelling), will be used to examine non-compliance measures (e.g., time spent using intervention, dropout rates) and the factors impacting it (e.g., symptom severity, comorbid mental health difficulties). GLMM analysis will be used to consider the clinician-supported BEeT subgroup and factors impacting change in the EDE-Q, such as treatment setting and clinician experience to determine if the main outcome is affected by the setting in which treatment is delivered. Descriptive analysis will investigate the embeddedness and implementation of the treatment based on the clinician and the patient questionnaires.

A within-trial economic evaluation will be conducted to determine the cost-effectiveness of clinician-supported BEeT compared to pure self-help BEeT and then to the WLC from a health system perspective using individual-level data. It is crucial that the cost-effectiveness study runs alongside the clinical trial to ensure the health economic evidence is directly related to the outcomes (patient experience, clinical outcomes and quality of life) 
and costs of study participants (who represent an eligible population in the real world) and can immediately be used by decision-makers in the translation of results into policy and practice (including scaling-up). A costutility framework which expresses the main outcomes as Quality Adjusted Life Years (QALYs) will be adopted. The main hypothesis is that the Incremental Cost Effectiveness Ratios (ICERs) are favourable of clinician-supported BEeT at 12 weeks i.e. ICER $<\$ 50,000$ per QALY gained. We will also assess whether the effect of clinician-supported $\mathrm{BEeT}$ is sustained at 3 months and re-calculate the ICERs. A utility-based and disease-generic quality of life measure, EQ-5D-5L, and a disease-specific measure, EDQOL, will be considered in the health economics analysis. Using a health system perspective, we will also be adopted to measure resource use (costs) and other benefits related to the participants and healthcare providers, including implementation costs such as clinician time costs to complete the training course and to deliver the programs. A cost questionnaire (database) will be developed specifically for this clinical trial. This questionnaire will be used to measure the type, amount and value of resources used (and hence adopting a "bottom up" costing approach). A secondary analysis will consider participants' need for additional technologies or equipment (e.g. laptop, tablet) and time costs for completing treatment sessions. Results will be presented as ICERs which will be calculated as the difference in total costs between clinician-supported compared to pure self-help $\mathrm{BEeT}$ (and then compared to WLC) divided by difference in QALYS. Sensitivity analysis will be undertaken.

For the main trial analysis, data will be analysed using IBM SPSS (Version 22.0.0.0, Chicago, IL).

\section{Discussion}

This trial is the first to examine the effectiveness, feasibility, and cost-effectiveness of a CBT-based, online selfhelp program in an Australian cohort of BN patients in real world healthcare settings. Should the results demonstrate the BEeT program to be an effective intervention, this research has the potential to change the treatment landscape for individuals with BN. Low-intensity programs, such as BEeT, are designed to be integrated within the early stages of a stepped-care model, either as a pure self-help intervention or a clinician-supported intervention $[23,73]$. By doing so, there can be improved resourcing in busy clinical services, more $\mathrm{BN}$ patients can receive treatment sooner, and hopefully result in a shorter duration of the illness and reduced burden to the individual and healthcare system.

There are several strengths of this trial. Firstly, the three-arm randomised controlled study design allows for assessment of the added benefits of clinician support in comparison to independent engagement with BEeT. Importantly, such outcomes must be considered when defining resource requirements for successful delivery of the intervention, for example the intensity of therapeutic support and necessary level of training of non-specialist clinicians. Secondly, the multisite design provides scope for implementation research through examination of the real-life application of a stepped care approach to EDs within the Australian healthcare system. Data will be collected from the perspective of participants, clinicians, and site managers to determine the site-specific variables affecting implementation of a supported self-help program across multiple outpatient clinics. Implementation science is a rapidly growing field of research, which is vital in facilitating the transfer of evidence-based interventions into clinical practice and community settings [74]. This is particularly important to consider within the domain of digital interventions, with research suggesting poor association between research studies on web-based interventions and meaningful outcomes for patients in clinical settings [75]. Thirdly, our trial includes an online training package for clinicians delivering the intervention, allowing for assessment of its capacity to upskill clinicians to guide patients through a self-help program without previous training in ED treatment.

There are limitations of this trial. To begin, the fidelity of the pure self-help intervention group may be compromised by the degree of contact between the researcher and participant as part of the disengagement protocol. This represents a potential confounding factor as such contact may limit the truly independent nature of the pure self-help condition. For example, follow up phone calls and emails made by researchers may create accountability for the participant to complete the sessions. To account for this in the statistical analyses, the frequency of contact initiated as part of the disengagement protocols will be recorded by research staff. Additionally, this trial started as the COVID-19 outbreak began globally in early to mid-2020. Due to the multisite design of the trial, the COVID-19 pandemic imposed operational challenges and significantly delayed recruitment. Equally, there were advantages of the digital delivery of this intervention in allowing for by-distance mental healthcare within a COVID-safe framework. Also, the mandatory GP medical screening of participants may form a barrier to participation for some individuals and could potentially impinge upon the key advantages of digital treatments, that being it's accessibility and anonymity. Finally, a high risk of bias may be present due to non-blinding of trial participants [76].

To conclude, this study attempts to address the need for innovative evidence-based digital interventions through the evaluation of a recently developed modern, online 
platform designed by an interdisciplinary team of digital programmers and designers as well as clinical staff [77]. It is hoped that this study contributes further to current knowledge regarding the ways in which we can effectively adopt an evidence-based mental health strategy which leverages innovative technology.

\section{Appendix A}

\section{List of Study Sites}

- Sydney Local Health District (SLHD)-Peter Beumont Eating Disorders Outpatient Service, Royal Prince Alfred Hospital, Camperdown NSW 2050.

- Northern Sydney Local Health District (NSLHD) Community Mental Health Service, Royal North Shore Hospital, St Leonards NSW 2065.

- Western NSW Local Health District (WNSWLHD) Community Mental Health Service, Orange NSW 2800.

- Charles Perkins Centre-Royal Prince Alfred Clinic (CPC-RPA Clinic) based at the University of Sydney, Camperdown NSW 2050.

- Headspace Liverpool, Liverpool NSW 2170.

- Headspace Wagga Wagga, Wagga Wagga NSW 2650.

- Headspace Camperdown, Camperdown NSW 2050.

- Community Links Wellbeing-ReFrame Program (Wollondilly and Wingecarribee).
Availability of data and materials

Not applicable.

\section{Declarations}

\section{Ethics approval and consent to participate}

This study has been approved by a Human Research Ethics Review Board (HREC \# X18-0486).

\section{Consent for publication \\ Not applicable.}

\section{Competing interests}

ST has received honoraria from the Takeda Group of Companies for commissioned reports, preparation of educational materials and public speaking engagements. He has also received both travel and research grants. He has also chaired their Clinical advisory Group for Binge Eating Disorders. ST has also received royalties for published book and/or book chapters from Hogrefe and Huber, McGraw Hill and Taylor and Francis. He is a member of the Technical Advisory Group for Eating Disorders established by the Commonwealth Department of Health and is also a member of the National Eating Disorders Collaboration (NEDC).

\section{Author details}

${ }^{1}$ InsideOut Institute, Central Clinical School, The University of Sydney | Sydney Local Health District, Sydney, Australia. ${ }^{2}$ School of Psychology, The University of Sydney, Sydney, Australia. ${ }^{3}$ Charles Perkins Centre, Faculty of Medicine and Health (Central Clinical School), The University of Sydney, Sydney, Australia. ${ }^{4}$ Sydney Local Health District Mental Health Services, Royal Prince Alfred Hospital, Sydney, Australia. ${ }^{5}$ Translational Health Research Institute, School of Medicine, Western Sydney University, Sydney, Australia. ${ }^{6}$ Camden and Campbelltown Hospitals, South Western Sydney Local Health District, Campbelltown, Australia. ${ }^{7}$ Sydney Institute for Women, Children and Their Families, Sydney Local Health District Camperdown, Camperdown, Australia. ${ }^{8}$ Sydney Health Economics Collaborative, Sydney Local Health District, Camperdown, Australia. ${ }^{9}$ Department of Psychological Medicine, Children's Hospital at Westmead, Sydney, Australia.

Received: 29 July 2021 Accepted: 21 September 2021

Published online: 14 October 2021

\section{References}

BN: Bulimia nervosa; ED: Eating disorder; CBT: Cognitive behavioural therapy; BEeT: Binge eating etherapy; WLC: Waitlist control; BMI: Body mass index; GLMM: Generalised linear mixed modelling; QALYs: Quality adjusted life years; ICERs: Incremental cost-effectiveness ratios.

\section{Acknowledgements}

The authors acknowledge the Sydney Local Health District for sponsoring this trial. The authors also acknowledge the five trial sites for participating in this project: Peter Beumont Eating Disorders Outpatient Service, Northern Sydney LHD, Western NSW LHD, Charles Perkins Centre-Royal Prince Alfred Clinic (CPCRPA Clinic), headspace Liverpool, headspace Camperdown, headspace Wagga Wagga, and Community Links Wellbeing ReFrame Program.

\section{Authors' contributions}

SM obtained funding for the trial and is the lead investigator. SB is the trial coordinator. SB, JMW, SM, and MC initiated the study design. SB led implementation at trial sites and initiation of recruitment and data collection. ST, DM, $J R, P H, S M, M C, S L$, and SM are named investigators on the grant funding this work. SL, MK and MC provided statistical expertise in clinical trial design and $\mathrm{SB}$ is conducting the primary statistical analysis. All authors contributed to the refinement of the study protocol. SB drafted the manuscript and all co-authors revised it and approved the final manuscript.

\section{Funding}

The study is funded by the NSW Health Translational Research Grants Scheme (TRGS). Funding is being managed by Dr. Sarah Maguire (co-author) who is appointed within the University of Sydney.
1. American Psychiatric Association. Diagnostic and statistical manual of mental disorders. 5th ed. Arlington: American Psychiatric Publishing; 2013.

2. Santomauro DF, Melen S, Mitchison D, Vos T, Whiteford H, Ferrari AJ. The hidden burden of eating disorders: an extension of estimates from the Global Burden of Disease Study 2019. Lancet Psychiatry. 2021:8(4):320-8.

3. Mitchison D, Hay P, Slewa-Younan S, Mond J. Time trends in population prevalence of eating disorder behaviors and their relationship to quality of life. PLoS ONE. 2012;7(11):e48450.

4. Mehler PS, Brown C. Anorexia nervosa-medical complications. J Eat Disord. 2015:3(1):1-8.

5. Jenkins PE, Hoste RR, Meyer C, Blissett JM. Eating disorders and quality of life: a review of the literature. Clin Psychol Rev. 2011:31(1):113-21.

6. Stice E, Marti CN, Rohde P. Prevalence, incidence, impairment, and course of the proposed DSM-5 eating disorder diagnoses in an 8-year prospective community study of young women. J Abnorm Psychol. 2013;122(2):445.

7. Bagaric M, Touyz S, Heriseanu A, Conti J, Hay P. Are bulimia nervosa and binge eating disorder increasing? Results of a population-based study of lifetime prevalence and lifetime prevalence by age in South Australia. Eur Eat Disord Rev. 2020;28(3):260-8.

8. Tannous WK, Hay P, Girosi F, Heriseanu Al, Ahmed MU, Touyz S. The economic cost of bulimia nervosa and binge eating disorder: a populationbased study. Psychol Med. 2021:17:1-5.

9. Evans EJ, Hay PJ, Mond J, Paxton SJ, Quirk F, Rodgers B, Jhajj AK, Sawoniewska MA. Barriers to help-seeking in young women with eating 
disorders: a qualitative exploration in a longitudinal community survey. Eat Disord. 2011;19(3):270-85.

10. Hamilton A, Mitchison D, Basten C, Byrne S, Goldstein M, Hay P, Heruc G, Thornton C, Touyz S. Understanding treatment delay: perceived barriers preventing treatment-seeking for eating disorders. Aust N Z J Psychiatry. 2021;12:00048674211020102.

11. Hay P, Chinn D, Forbes D, Madden S, Newton R, Sugenor L, Touyz S, Ward W. Royal Australian and New Zealand College of Psychiatrists clinical practice guidelines for the treatment of eating disorders. Aust N Z J Psychiatry. 2014;48(11):977-1008.

12. Fairburn CG, Jones R, Peveler RC, Carr SJ, Solomon RA, O'Connor ME, Burton J, Hope RA. Three psychological treatments for bulimia nervosa: a comparative trial. Arch Gen Psychiatry. 1991;48(5):463-9.

13. Agras WS, Crow SJ, Halmi KA, Mitchell JE, Wilson GT, Kraemer HC. Outcome predictors for the cognitive behavior treatment of bulimia nervosa: data from a multisite study. Am J Psychiatry. 2000;157(8):1302-8.

14. Collaboration NED. Eating disorders prevention, treatment and management: an evidence review. Sydney: NEDC; 2010.

15. Hart LM, Granillo MT, Jorm AF, Paxton SJ. Unmet need for treatment in the eating disorders: a systematic review of eating disorder specific treatment seeking among community cases. Clin Psychol Rev. 2011;31(5):727-35.

16. Bauer S, Moessner M. Harnessing the power of technology for the treatment and prevention of eating disorders. Int J Eat Disord. 2013:46(5):508-15.

17. Fairburn CG. Cognitive behavior therapy and eating disorders. New York: Guilford Press; 2008

18. National Eating Disorders Collaboration (NEDC). Consultation Papers. Canberra: National Eating Disorders Collaboration; 2014.

19. Pellizzer ML, Waller G, Wade TD. A pragmatic effectiveness study of 10-session cognitive behavioural therapy (CBT-T) for eating disorders: targeting barriers to treatment provision. Eur Eat Disord Rev. 2019;27(5):557-70.

20. Paxton SJ, Hay P, Touyz SW, Forbes D, Madden S, Girosi F, Doherty A, Cook L, Morgan C. Paying the Price: The economic and social impact of eating disorders in Australia. 2012 Commissioned by The Butterfly Foundation. NSW, Australia.

21. Mental Health and Suicide Prevention - Interim Report. Prepared by: Select Committee on Mental Health and Suicide Prevention, Commonwealth of Australia. 2021.

22. Ljotsson B, Lundin C, Mitsell K, Carlbring P, Ramklint M, Ghaderi A. Remote treatment of bulimia nervosa and binge eating disorder: a randomized trial of Internet-assisted cognitive behavioural therapy. Behav Res Ther. 2007:45(4):649-61.

23. Bower P, Gilbody S. Stepped care in psychological therapies: access, effectiveness and efficiency: narrative literature review. Br J Psychiatry. 2005;186(1):11-7.

24. Zabinski MF, Wilfley DE, Pung MA, Winzelberg AJ, Eldredge K, Taylor CB. An interactive internet-based intervention for women at risk of eating disorders: a pilot study. Int J Eat Disord. 2001;30(2):129-37.

25. Fisk M, Livingstone A, Pit SW. Telehealth in the context of COVID-19: changing perspectives in Australia, the United Kingdom, and the United States. J Med Internet Res. 2020;22(6):e19264.

26. Figueroa CA, Aguilera A. The need for a mental health technology revolution in the COVID-19 pandemic. Front Psychiatry. 2020;11.

27. Torous J, Myrick KJ, Rauseo-Ricupero N, Firth J. Digital mental health and COVID-19: using technology today to accelerate the curve on access and quality tomorrow. JMIR Mental Health. 2020;7(3):e18848.

28. Taylor CB, Fitzsimmons-Craft EE, Graham AK. Digital technology can revolutionize mental health services delivery: the COVID-19 crisis as a catalyst for change. Int J Eat Disord. 2020;53(7):1155-7.

29. Jain N, Jayaram M. Comment on" Digital Mental Health and COVID-19: Using Technology Today to Accelerate the Curve on Access and Quality Tomorrow". JMIR Mental Health. 2020;7(8):e23023.

30. Touyz S, Lacey H, Hay P. Eating disorders in the time of COVID-19. J Eat Disord. 2020;8(19).

31. Weissman RS, Bauer S, Thomas JJ. Access to evidence-based care for eating disorders during the COVID-19 crisis. 2020;55:639-646.

32. Fernández-Aranda F, Casas M, Claes L, Bryan DC, Favaro A, Granero R, Gudiol C, Jiménez-Murcia S, Karwautz A, Le Grange D, Menchón JM. COVID-19 and implications for eating disorders. Eur Eat Disord Rev. 2020:28(3):239.
33. Aardoom JJ, Dingemans AE, Spinhoven P, Van Furth EF. Treating eating disorders over the internet: a systematic review and future research directions. Int J Eat Disord. 2013:46(6):539-52.

34. Anastasiadou D, Folkvord F, Lupiañez-Villanueva F. A systematic review of mHealth interventions for the support of eating disorders. Eur Eat Disord Rev. 2018;26(5):394-416.

35. Barakat S, Maguire S, Smith KE, Mason TB, Crosby RD, Touyz S. Evaluating the role of digital intervention design in treatment outcomes and adherence to eTherapy programs for eating disorders: a systematic review and meta-analysis. Int J Eat Disord. 2019;52(10):1077-94.

36. Dölemeyer R, Tietjen A, Kersting A, Wagner B. Internet-based interventions for eating disorders in adults: a systematic review. BMC Psychiatry. 2013;13(1):1-6.

37. Hay PJ, Claudino AM. Bulimia nervosa: online interventions. BMJ Clin Evid. 2015:3:1-16.

38. Loucas CE, Fairburn CG, Whittington C, Pennant ME, Stockton S, Kendall T. E-therapy in the treatment and prevention of eating disorders: a systematic review and meta-analysis. Behav Res Ther. 2014;1 (63):122-31.

39. Melioli T, Bauer S, Franko DL, Moessner M, Ozer F, Chabrol H, Rodgers RF. Reducing eating disorder symptoms and risk factors using the internet: $a$ meta-analytic review. Int J Eat Disord. 2016;49(1):19-31.

40. Hay PP, Bacaltchuk J, Stefano S, Kashyap P. Psychological treatments for bulimia nervosa and binging. Cochrane Database Syst Rev. 2009(4).

41. Wilson GT, Zandberg LJ. Cognitive-behavioral guided self-help for eating disorders: effectiveness and scalability. Clin Psychol Rev. 2012;32(4):343-57.

42. Thiels C, Schmidt U, Troop N, Treasure J, Garthe R. Binge frequency predicts outcome in guided self-care treatment of bulimia nervosa. Eur Eat Disord Rev. 2000;8(4):272-8.

43. Baumeister $H$, Reichler $L$, Munzinger $M$, Lin J. The impact of guidance on Internet-based mental health interventions-a systematic review. Internet Interv. 2014;1(4):205-15.

44. Linardon J, Hindle A, Brennan L. Dropout from cognitive- behavioral therapy for eating disorders: a meta-analysis of randomized, controlled trials. Int J Eat Disord. 2018;51(5):381-91. https://doi.org/10.1002/eat. 22850.

45. Sánchez-Ortiz VC, Munro C, Stahl D, House J, Startup H, Treasure J, Williams C, Schmidt U. A randomized controlled trial of internet-based cognitive-behavioural therapy for bulimia nervosa or related disorders in a student population. Psychol Med. 2011;41(2):407.

46. Hay PP, Bacaltchuk J, Stefano S. Psychotherapy for bulimia nervosa and binging. Cochrane Database Syst Rev. 2004(3).

47. Treasure J, Schmidt U, Troop N, Tiller J, Todd G, Turnbull S. Sequential treatment for bulimia nervosa incorporating a self-care manual. Br J Psychiatry. 1996;168(1):94-8.

48. Pretorius N, Arcelus J, Beecham J, Dawson H, Doherty F, Eisler I, Gallagher C, Gowers S, Isaacs G, Johnson-Sabine E, Jones A. Cognitive-behavioural therapy for adolescents with bulimic symptomatology: the acceptability and effectiveness of internet-based delivery. Behav Res Ther. 2009:47(9):729-36.

49. Barakat S, Maguire S, Surgenor L, Donnelly B, Miceska B, Fromholtz K, Russell J, Hay P, Touyz $S$. The role of regular eating and self-monitoring in the treatment of bulimia nervosa: a pilot study of an online guided self-help CBT program. Behav Sci. 2017;7(3):39.

50. Torous J, Roberts LW. Needed innovation in digital health and smartphone applications for mental health: transparency and trust. JAMA Psychiat. 2017;74(5):437-8.

51. Fairburn CG, Rothwell ER. Apps and eating disorders: a systematic clinical appraisal. Int J Eat Disord. 2015;48(7):1038-46.

52. Chan AW, Tetzlaff JM, Altman DG, Laupacis A, Gøtzsche PC, Krleža-Jerić K, Hróbjartsson A, Mann H, Dickersin K, Berlin JA, Doré CJ. SPIRIT 2013 statement: defining standard protocol items for clinical trials. Ann Intern Med. 2013;158(3):200-7.

53. Fairburn CG, Beglin SJ. Eating disorder examination questionnaire (6.0). In: Fairburn CG, editor. Cognitive behavior therapy and eating disorders. New York: Guildford Press; 2008. pp. 309-315.

54. Peterson CB, Crosby RD, Wonderlich SA, Joiner T, Crow SJ, Mitchell JE, Bardone-Cone AM, Klein M, Le Grange D. Psychometric properties of the eating disorder examination-questionnaire: Factor structure and internal consistency. Int J Eat Disord. 2007;40:386-9. 
55. Gideon N, Hawkes N, Mond J, Saunders R, Tchanturia K, Serpell L. Development and psychometric validation of the EDE-QS, a 12 item short form of the Eating Disorder Examination Questionnaire (EDE-Q). PLoS ONE. 2016;11(5):e0152744.

56. Prnjak K, Mitchison D, Griffiths S, Mond J, Gideon N, Serpell L, Hay P. Further development of the 12-item EDE-QS: identifying a cut-off for screening purposes. BMC Psychiatry. 2020;20(1):1-7.

57. Engel S, Wittrock D, Crosby R, Wonderlich S, Mitchell J, Kolotkin R. Development and psychometric validation of an eating disorder-specific health-related quality of life instrument. Int J Eat Disord. 2006;39:62-71.

58. Stunkard AJ, Messick S. The three-factor eating questionnaire to measure dietary restraint, disinhibition and hunger. J Psychosom Res. 1985:29:71-83.

59. Kessler RC, Andrews G, Colpe LJ, Hiripi E, Mroczek DK, Normand SL, Walters EE, Zaslavsky AM. Short screening scales to monitor population prevalences and trends in non-specific psychological distress. Psychol Med. 2002;32:959-76.

60. Staples LG, Dear BF, Gandy M, Fogliati V, Fogliati R, Karin E, Nielssen O, Titov N. Psychometric properties and clinical utility of brief measures of depression, anxiety, and general distress: the PHQ-2, GAD-2, and K-6. Gen Hosp Psychiatry. 2019;1 (56):13-8.

61. Merson F, Newby J, Shires A, Millard M, Mahoney A. The temporal stability of the Kessler psychological distress scale. Aust Psychol. 2021;56(1):38-45.

62. Stolk Y, Kaplan I, Szwarc J. Clinical use of the Kessler psychological distress scales with culturally diverse groups. Int J Methods Psychiatr Res. 2014;23(2):161-83.

63. Young JE, Brown G. Young schema questionnaire-short form; Version 3. Psychological Assessment. 2005.

64. Williams A, EuroQol G. EuroQol - a new facility for the measurement of health-related quality of life. Health Policy. 1990;16(3):199-208.

65. Hatcher RL, Gillaspy JA. Development and validation of a revised short version of the Working Alliance Inventory. Psychother Res. 2006;16(1):12-25.

66. Munder T, Wilmers F, Leonhart R, Linster HW, Barth J. Working Alliance Inventory-Short Revised (WAI-SR): psychometric properties in outpatients and inpatients. Clin Psychol Psychother Int J Theory Pract. 2010;17(3):231-9.

67. Herrero R, Vara M, Miragall M, Botella C, García-Palacios A, Riper H, Kleiboer A, Baños RM. Working alliance inventory for online interventionsshort form (Wai-tech-sf): the role of the therapeutic alliance between patient and online program in therapeutic outcomes. Int J Environ Res Public Health. 2020;17(17):6169.
68. Peters DH, Adam T, Alonge O, Agyepong IA, Tran N. Implementation research: what it is and how to do it. BMJ. 2013;20:347.

69. Aardoom JJ, Dingemans AE, Spinhoven P, van Ginkel JR, de Rooij M, van Furth EF. Web-based fully automated self-help with different levels of therapist support for individuals with eating disorder symptoms: a randomized controlled trial. J Med Internet Res. 2016;18(6):e159.

70. de Zwaan M, Herpertz S, Zipfel S, Svaldi J, Friederich HC, Schmidt F, Mayr A, Lam T, Schade-Brittinger C, Hilbert A. Effect of internet-based guided self-help vs individual face-to-face treatment on full or subsyndromal binge eating disorder in overweight or obese patients: the INTERBED randomized clinical trial. JAMA Psychiat. 2017;74(10):987-95.

71. ter Huurne ED, de Haan HA, Postel MG, van der Palen J, VanDerNagel JE, DeJong CA. Web-based cognitive behavioral therapy for female patients with eating disorders: randomized controlled trial. J Med Internet Res. 2015;17(6):e152.

72. StataCorp. . Stata statistical software: release 15. College Station: StataCorp LLC; 2017.

73. Bauer S, Okon E, Meermann R, Kordy H. Technology-enhanced maintenance of treatment gains in eating disorders: efficacy of an intervention delivered via text messaging. J Consult Clin Psychol. 2012;80(4):700.

74. LaMonica HM, Davenport TA, Braunstein K, Ottavio A, Piper S, Martin C, Hickie IB, Cross S. Technology-enabled person-centered mental health services reform: strategy for implementation science. JMIR Mental Health. 2019;6(9):e14719.

75. Glasgow RE, Lichtenstein E, Marcus AC. Why don't we see more translation of health promotion research to practice? Rethinking the efficacy-toeffectiveness transition. Am J Public Health. 2003;93(8):1261-7.

76. Schünemann H, Brożek J, Guyatt G, Oxman A, editors. GRADE handbook for grading quality of evidence and strength of recommendations. Updated October 2013. The GRADE Working Group, 2013. http://www. guidelinedevelopment.org/handbook.

77. Graham AK, Wildes JE, Reddy M, Munson SA, Barr Taylor C, Mohr DC. Usercentered design for technology-enabled services for eating disorders. Int J Eat Disord. 2019;52(10):1095-107.

\section{Publisher's Note}

Springer Nature remains neutral with regard to jurisdictional claims in published maps and institutional affiliations.
Ready to submit your research? Choose BMC and benefit from:

- fast, convenient online submission

- thorough peer review by experienced researchers in your field

- rapid publication on acceptance

- support for research data, including large and complex data types

- gold Open Access which fosters wider collaboration and increased citations

- maximum visibility for your research: over $100 \mathrm{M}$ website views per year

At BMC, research is always in progress.

Learn more biomedcentral.com/submissions 